\title{
The effects of separate and combined perirhinal and prefrontal cortex lesions on spatial memory tasks in the rat
}

\author{
DAVID K. BILKEY and PING LIU \\ University of Otago, Dunedin, New Zealand
}

\begin{abstract}
In order to compare the relative involvement of prefrontal and temporal regions in spatial memory, rats with bilateral electrolytic lesions of perirhinal cortex, prefrontal cortex, or both regions were tested in the water and radial-arm maze. Perirhinal and prefrontal lesions produced similar performance deficits during water-maze acquisition and in the radial-maze procedure. Perirhinal cortex lesions also disrupted performance in a water-maze probe trial conducted 5 min after four training trials, whereas prefrontal lesions did not. Surprisingly, the combined-lesion group was not impaired during water-maze acquisition and performed significantly better than the perirhinal-lesioned group in the probe trial. This finding is interpreted in terms of the executive function of prefrontal cortex and its possible role in switching behavior between different search strategies, only some of which may be dependent on perirhinal cortex.
\end{abstract}

The results of a number of recent studies indicate that the perirhinal cortex region of the temporal cortex contributes to normal learning and memory. Lesions of the perirhinal cortex have been shown to impair memory performance in delayed nonmatching-to-sample tasks in both primates and rodents (see, e.g., Buckley, Gaffan, \& Murray, 1997; Meunier, Bachevalier, Mishkin, \& Murray, 1993; Mumby \& Pinel, 1994; Murray, 1996; Wiig \& Bilkey, 1995; Zola-Morgan, Squire, Amaral, \& Suzuki, 1989), and several recent findings suggest that perirhinal cortex lesions also disturb the performance of spatial tasks. For example, rats with bilateral lesions confined to the perirhinal cortex displayed deficits in both spatial reference and working memory versions of the radial-arm maze task (Liu \& Bilkey, 1998b), a delay-dependent deficit in a delayed nonmatching-to-position procedure conducted in the T maze (Liu \& Bilkey, 1998a; Wiig \& Bilkey, 1994b; although see Ennaceur, Neave, \& Aggleton, 1996), and acquisition and retention deficits in a spatial reference memory task conducted in the water maze (Liu \& Bilkey, 1998c; Wiig \& Bilkey, 1994a).

Several other brain regions have been implicated in mnemonic processes, and a variety of evidence points to a role for the prefrontal cortex (e.g., Goldman-Rakic, 1995; Shimamura, 1995). Prefrontal lesions have long been associated with memory dysfunction, and it has been pro-

This research was supported by a grant from the New Zealand Lottery Grants Board and the Health Research Council to D.K.B. We thank Barry Dingwall, Chris Perk, Lindsay Robertson, Brendon Sturgeon, Matthew Johnston, Rachel Kyd, and Sara-Lee Illingworth for their help. Correspondence should be addressed to D. K. Bilkey, Department of Psychology and the Neuroscience Research Centre, University of Otago, P. O. Box 56, Dunedin, New Zealand (e-mail: sycodkb@otago.ac.nz). posed that this region is involved in working memorythat is, the process by which information is kept active or "on line" during a memory task (Baddeley, 1986). It has been shown, for example, that neurons in the prefrontal cortex are active during working-memory tasks (e.g., Funahashi, Inoue, \& Kubota, 1997; Goldman-Rakic, 1995; Miller, Erickson, \& Desimone, 1996). Furthermore, lesions of the prefrontal cortex produce working-memory deficits in both primates and rats (see, e.g., Goldman \& Rosvold, 1970; Granon, Vidal, Thinus-Blanc, Changeux, \& Poucet, 1994; Sanchez-Santed, Bruin, Heinsbroek, \& Verwer, 1997; Shaw \& Aggleton, 1993; Shimamura, 1995).

Since working-memory processes may involve the activation and manipulation of representations that are dependent on the temporal cortex, it is likely that prefrontaltemporal interactions are critical to the normal functioning of this system. Whereas this hypothesis has previously been studied in primates (Fuster, Bauer, \& Jervey, 1985; Gutnikov, Ma, \& Gaffan, 1997; Parker \& Gaffan, 1998), little is known about temporal-prefrontal processes in rats. The purpose of the present study, therefore, is to directly compare the effects of lesions of the prefrontal and perirhinal cortex on several tasks that are commonly used to test spatial memory in rats and, critically, to investigate the effects of concurrent lesions made to both of these structures. If a primary function of the prefrontal cortex involves accessing representations that may, in part, be perirhinal dependent, one would expect that combined lesions in these two structures should either have no additional effect (if one lesion disrupts the whole system), or an additive effect (if a single lesion is only partially disruptive), when compared with the deficit produced by a lesion of either structure alone. 


\section{METHOD}

\section{Subjects}

Male Sprague-Dawley rats, weighing between 350 and $400 \mathrm{~g}$ at the time of surgery, were housed individually in wire mesh cages and maintained on a 12:12-h light:dark cycle. Food and water were freely available presurgery and throughout the water-maze experiment. Rats were food deprived, until they were $85 \%$ of their freefeeding body weight, during the radial-arm maze experiment. Surgical and behavioral procedures were conducted during the light cycle.

\section{Surgery}

Fifty-one rats were randomly divided into four groups, anesthetized with sodium pentobarbital $(50 \mathrm{mg} / \mathrm{kg}$, i.p.), and placed in a stereotaxic apparatus. A midline incision was made and the scalp retracted to expose the skull. Perirhinal cortex lesions (PRC, $n=15$ ) were created by inserting electrodes into the brain through trephines drilled at the coordinates $4.5,5.5,6.5 \mathrm{~mm}$ posterior to Bregma (AP), and $5.5 \mathrm{~mm}$ lateral to the midline (L). Monopolar electrodes, which were constructed of $125-\mu$ m-diameter Teflon-coated wire were orientated laterally at $10^{\circ}$ from the vertical and lowered to a depth (D) of 4.5-5.0 $\mathrm{mm}$ from the dural surface. There has been some debate in the literature as to which area in the rat brain is homologous to the primate prefrontal region. We treated the anterior cingulate (see Kolb, 1990; Zilles's Cg1, Cg2, and Cg3) as an equivalent to at least a portion of the primate prefrontal cortex that receives afferents from the medialis dorsalis of the thalamus. Prefrontal cortex lesions (PFC, $n=12$ ) were made by inserting electrodes at the following coordinates: AP $+2.0 \mathrm{~mm}, \mathrm{~L} 1.6 \mathrm{~mm}, \mathrm{D} 2.0$ $\mathrm{mm}$; AP $+3.0 \mathrm{~mm}, \mathrm{~L} 1.6 \mathrm{~mm}, \mathrm{D} 1.3$ and $2.1 \mathrm{~mm}$; AP $+4.5 \mathrm{~mm}$, $\mathrm{L} 1.6 \mathrm{~mm}$, D $1.0 \mathrm{~mm}$; angled $16^{\circ}$ toward the midline. Combined perirhinal-prefrontal cortex lesions (PRPF, $n=12$ ) were created by sequential lesioning at both the above sets of coordinates. The prefrontal and perirhinal coordinates were derived from Paxinos and Watson (1986), and the perirhinal cortex boundaries were as described by Burwell, Witter, and Amaral (1995). Lesions were induced by passing dc current at $2 \mathrm{~mA}$ through the electrodes for a duration of $8 \mathrm{sec}$ at each position. Sham rats $(n=12)$ were operated on in the same manner as the experimental groups except that electrodes were not lowered into the brain. Rat body temperature was maintained at $37^{\circ} \mathrm{C}$ throughout the surgical period. Once these procedures were complete, the wound was sutured, and the rats were kept warm and were monitored until spontaneous movement occurred. They were then returned to their home cages to recover for 10 days prior to behavioral testing.

\section{Apparatus and Training Procedure}

Experiment 1: Morris water-maze task. The water-maze pool was a black circular tank measuring $150 \mathrm{~cm}$ in diameter and $36 \mathrm{~cm}$ in height. It was filied with water to a depth of approximately $24 \mathrm{~cm}$ and maintained at a temperature of $26^{\circ} \pm 1^{\circ} \mathrm{C}$. Four points around the edge of the pool were designated as north, south, east, and west, which allowed the pool to be divided into four corresponding quadrants (NE, SW, NW, and SE). The locations of extramaze cues, such as laboratory furniture and lights, were held constant throughout the experiment. A video camera was mounted above the center of the pool, and animal behavior was recorded throughout the experimental period.

Place navigation (Day 1-Day 5). A black platform $(10 \times 10 \mathrm{~cm})$, submerged $1.0 \mathrm{~cm}$ below the surface of the water, was placed in the center of the SE quadrant. The rat was placed into the pool facing toward the wall and was allowed to swim in search of the platform. If the platform was located, the rat was permitted to remain on it for $10 \mathrm{sec}$ before being removed and placed in a holding box. If the rat could not find the platform within $120 \mathrm{sec}$, it was placed onto it for $10 \mathrm{sec}$ before being returned to the holding box. All rats received eight trials per day for 5 consecutive days with a 2-min intertrial in- terval. Starting locations (N, S, W, E) were pseudorandomly selected without replacement within each block of four trials.

Probe test (Day 6-Day 7). On each day of probe testing, each rat was given four training trials as described above. After the completion of the fourth training trial, the rat was removed from the platform and placed in the holding box for a delay period of either 5 or $300 \mathrm{sec}$. During this delay, the platform was removed from the pool. At the end of the delay period, the rat was reintroduced into the pool at the starting location $\mathrm{N}$ for a 1 -min probe trial during which it was allowed to swim freely. At the end of the probe trial, the rat was removed from the pool, dried, and returned to its home cage until the next day of testing. Delay order was counterbalanced across rats and groups so that each rat was tested once at each delay.

Reversal training (Day 8 -Day 9). On Day 8, the hidden platform was shifted to the center of the NW quadrant. All rats received eight trials per day for 2 days with a 2 -min intertrial interval.

Behavioral measures. After completing the experiment, we analyzed several performance variables taken from the videotape recordings. Swimming speed was analyzed on the first training trial of Day I and latency to reach the platform was measured during the place navigation and reversal training trials. The length of the path taken from the release point to the platform was determined for each animal on the eight training trials conducted on the final day of water maze training (Day 5). A path deviation measure was also determined on Day 5 by summing the distance between 10 equally spaced points placed on the actual path and a straight virtual path that directly linked the start and target points. Finally, the angle of approach to the platform was calculated by fitting a line to the path of the rat from the position where it approached to within $20 \mathrm{~cm}$ of the platform and the point at which it finally made contact with the platform. The angles of approach to the platform were then analyzed using circular statistics as described by Zar (1996), whereby for each animal, a vector was generated that described the mean approach angle over the eight trials. The length of this vector provided a measure of the animal's tendency to approach the platform from some preferred direction. The percentage of time spent in each quadrant and the latency to reach the position where the platform had previously been located were analyzed using the probe trial data.

Experiment 2: Radial-arm maze task. The maze was constructed of clear Plexiglas with a $34-\mathrm{cm}$ diameter central platform and eight $70-\mathrm{cm}$ long, $9-\mathrm{cm}$ wide arms with $6-\mathrm{cm}$ high walls. A Plexiglas food cup was located at the end of each arm, and the maze was pivoted so that it could be rotated around the central axis. The maze was located in the same room as the water maze, and extramaze cues were held constant throughout the experiment. Breakfast cereal was used as a reinforcer.

All rats were trained in a standard working memory task (Olton \& Samuelson, 1976). On the first 2 days, rats were placed onto the central platform and were allowed to explore the maze for $20 \mathrm{~min}$, during which time reinforcers were scattered at various distances along the arms. For the following 8 days, one reinforcer was placed at the end of each arm and the rat was allowed to choose freely between arms. Each trial was running until: (1) all eight arms had been chosen, (2) $10 \mathrm{~min}$ had elapsed since the start of the test, or (3) $2 \mathrm{~min}$ had elapsed since the rat's last choice (Olton, Walker, \& Gage, 1978). Each rat received one trial per day. The maze was rotated and wiped clean after each trial.

Behavioral measures. Performance was measured by counting the number of entries that rats made into arms that had been visited previously (errors). An arm entry was scored when the whole body (excluding the tail) of the rat was inside the respective arm.

\section{Histological Procedure}

On completion of the behavioral experiments, the rats were perfused with $10 \%$ formalin solution in $0.9 \%$ saline. The brains were removed and immersed in $10 \%$ formalin solution for 1 day and then switched to a $30 \%$ sucrose-formalin solution for 3-5 days. Each brain 
was sectioned $(60 \mu \mathrm{m})$ in the coronal plane on a cryostat, mounted on slides, and stained with thionin. Coronal sections were selected from each animal at the following locations: PFC: $+1.0,+2.0,+3.0$, +4.0 , and $+5.0 \mathrm{~mm}$; PRC: $-3.5,-4.5,-5.5,-6.5$, and $-7.5 \mathrm{~mm}$. Each section was then analyzed in order to obtain the smallest and largest lesions.

\section{RESULTS}

\section{Histological Results}

Prefrontal cortex lesions. The rats in the PFC group had extensive bilateral damage to cingulate cortical area 1 and 3 , with, in some rats, sparing of the most anterior and posterior portions. There was involvement of cingulate area 2 in a few rats. All rats had damage to frontal area 2 and, in some rats, lesions involved a portion of frontal area 1 (Figure 1A). There was no evidence of damage to lateral orbital cortex or forceps minor corpus callosum.

Perirhinal cortex lesions. Histological examination revealed that all PRC group rats had received bilateral damage to the perirhinal cortex as described previously (Liu \& Bilkey, 1998b, 1998c). In 11 of 15 animals, nearly complete lesions of the middle to posterior portions of the perirhinal cortex were obtained, with some sparing of anterior regions. In the remaining 4 rats, the lesions also included more posterior portions of the perirhinal cortex. Most perirhinal cortical lesions extended from layer I to layer V, terminating at the level of the external capsule. There was no evidence of damage to the temporal cortex, ventral hippocampus, or lateral entorhinal cortex. There was minimal damage to the most anterior portions of postrhinal cortex. The location and extent of the smallest and largest perirhinal cortical lesions are presented in Figure 1B.

Combined perirhinal-prefrontal cortex lesions. The histological analysis indicated that the rats in the PRPF group had received bilateral perirhinal and prefrontal cortical damage that was a combination of the separate lesions described above. There was also no evidence of damage to the temporal cortex, ventral hippocampus, lateral entorhinal cortex, lateral orbital cortex, or forceps minor corpus callosum. Figure 1C illustrates the location and extent of the smallest and largest perirhinal and prefrontal cortical lesions.

\section{Behavioral Results: \\ Experiment 1. Morris Water-Maze Task}

Place navigation. The latency to find the hidden platform decreased rapidly during the first few days of training, and performance was near asymptote by the 4th day (Figure 2). All three lesioned groups had longer latencies during the initial training compared with latencies of control rats (Figure 2). There was a significant group $[F(3,47)=2.80, p=.05]$ and day $[F(4,188)=85.43, p<$ $.0001]$ effect in the analysis of variance (ANOVA), but no group $\times$ day interaction $[F(12,188)=1.15, p=.32]$. Subsequent post hoc analysis (Fisher's LSD test) showed that on Day 1, the sham group had significantly shorter latencies than did the PRC $(p<.05)$ and PFC $(p<.01)$ groups but not the PRPF group, whereas the PFC group had significantly longer latencies than the PRPF $(p<.01)$ but not the PRC group. On Day 2, there were significant differences between only the PFC and SHAM groups $(p<.01)$, and from Days 3 to 5 there were no significant differences between any of the groups. No difference was found between any of the groups when swimming speeds $[F(3,47)=1.11, p=.35]$ and latencies $[F(3,47)=1.64$, $p=.19]$ were compared on the first training trial of Day 1 .

In order to test whether lesioned animals displayed evidence of stereotyped behavior, a comparison of the mean path length and path deviation generated over the eight trials of Day 5 were compared. It was assumed that if lesioned animals had adopted a stereotyped approach strategy (e.g., always approaching the platform from one direction), these measures would be higher. However, an ANOVA revealed that there were no significant differences between groups for either path length $[F(3,47)=$ $2.55, p=.07]$ or path deviation $[F(3,47)=1.29, p=.29]$. A further ANOVA of the length of the vector that described the individual animal's tendency to approach the platform from some preferred direction indicated that there was no significant difference $[F(3,47)=0.12, p=.95]$ between any of the groups.

Probe test. The results of a two-way ANOVA revealed that there was a delay effect $[F(1,47)=7.00, p=.01]$, but no significant group effect $[F(3,47)=1.20, p=.32]$ or group $\times$ delay interaction $[F(3,47)=0.63, p=.59]$ in terms of the latency to the previous platform location. When the percentage of time in the target quadrant was analyzed, there was a significant group effect $[F(3,47)=3.26, p<$ $.03]$, but no significant delay effect $[F(1,47)=1.38, p=$ $.25]$ or group $\times$ delay interaction $[F(3,47)=1.21, p=.32]$ (Figure 3). Because previous studies from our lab had shown that PRC lesions produce a delay-dependent effect on probe trials conducted several minutes after a training trial, a multiple comparisons test (Fisher's LSD) was conducted on these data. This revealed that the performance of the PRPF animals was significantly better than that of the PRC $(p<.05)$ and PFC $(p<.01)$ groups at the 5-sec delay, but that there was no difference between the SHAM/ PRC, SHAM/PFC, SHAM/PRPF, and PRC/PFC groups. At the 300-sec delay, the PRC group spent significantly less time in the correct quadrant than did the SHAM $(p<.05), \operatorname{PFC}(p<.05)$, and PRPF $(p<.01)$ groups. There was no significant difference between any of the other groups. When the data from all groups were analyzed, there was no significant correlation between the latency to reach the previous platform location and the percentage of time spent in the target quadrant during the 300 -sec delay probe $(r=.09, p=.75)$.

Reversal training. All groups showed an initial increase and then a rapid decrease in escape latency during the reversal procedure during which the hidden platform was relocated to the opposite quadrant. Figure 4 illustrates the mean scores for each block of four trials across the 2 days of training. In the overall ANOVA, there was a significant block effect $[F(3,141)=52.95, p<$ $.0001]$ and a group $\times$ block interaction $[F(9,141)=2.07$, 

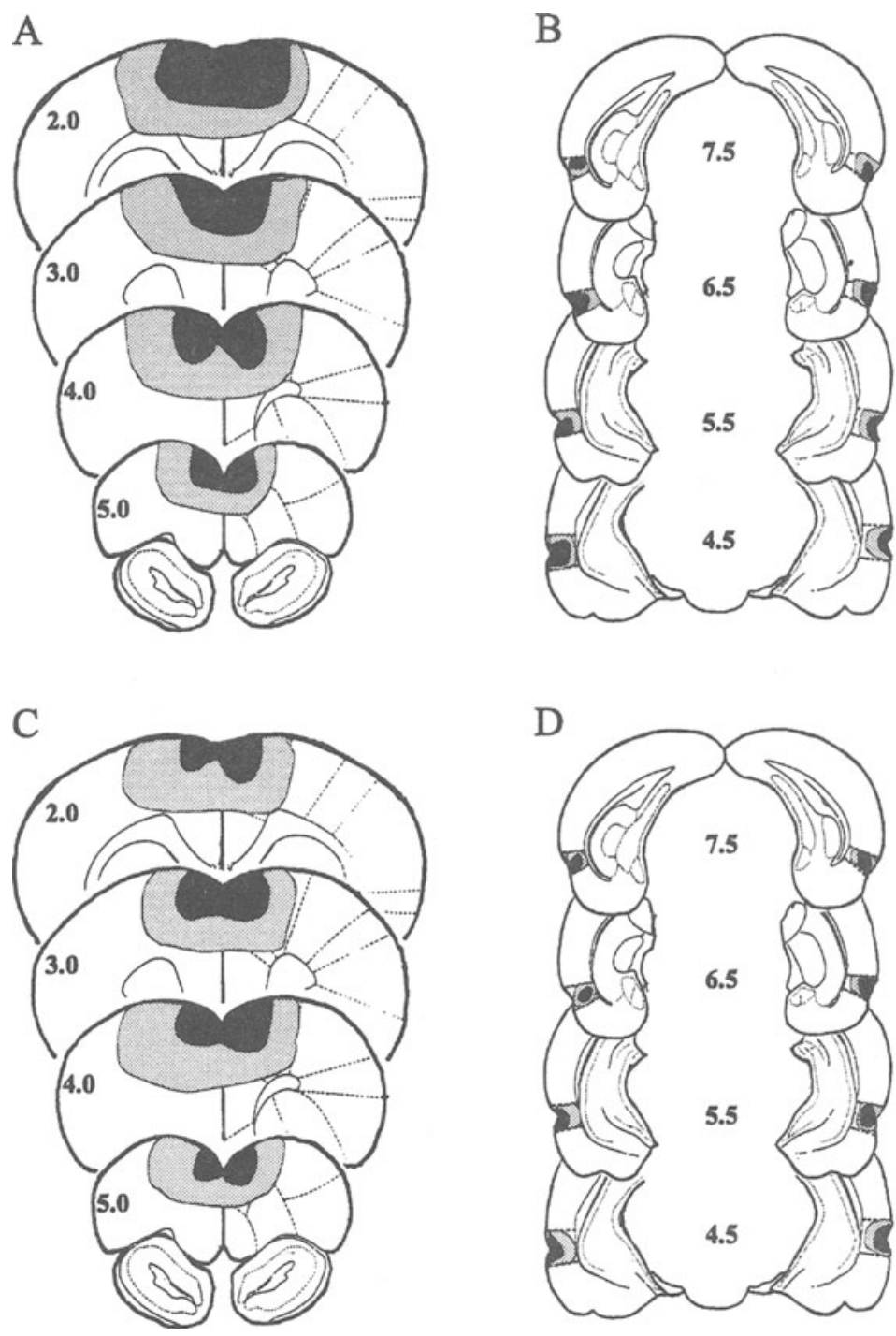

Figure 1. The location and extent of the smallest (filled) and largest (stippled) electrolytic lesions of prefrontal (A), perirhinal (B), and combined prefrontal-perirhinal cortices ( $C$ and $D$ ) in coronal sections. Numbers represent the distance in millimeters to bregma.

$p=.036]$, but no group effect $[F(3,47)=2.45, p=.075]$. A Fisher's LSD post hoc test revealed that during the first block of reversal training the SHAM group had significantly shorter latencies than did the PRC $(p<.01)$, PFC $(p<.05)$, and PRPF $(p<.01)$ groups. The PRPF group also performed significantly worse than both the PRC $(p<.05)$ and PFC $(p<.05)$ groups. There were no significant differences between any groups on Blocks 2-4.

\section{Behavioral Results:}

\section{Experiment 2. Radial-Arm Maze Task}

All lesioned groups made significantly more errors during the acquisition of the radial maze task (Figure 5). An ANOVA revealed that there was a significant effect of group $[F(3,46)=17.42, p<.0001]$ and day $[F(7,322)=$ $5.10, p<.0001]$, but there was no group $\times$ day inter- action $[F(21,322)=0.99, p=.46]$. A post hoc Fisher's LSD test revealed that all lesioned rats made significantly more errors than did the sham controls during the first 5 days of training (all $p s<.01$ ). On Day 6 , there were significant differences between SHAM/PRC and SHAM/ PFC $(p<.05)$, but not between the SHAM/PRPF, PRC/ PRPF, and PFC/PRPF groups. On Day 7 , significant differences were only observed between the SHAM/PFC $(p<.01)$ and PFC/PRC $(p<.05)$ groups, and there were no significant differences between any groups on Day 8 .

\section{DISCUSSION}

The findings of the present study demonstrate that rats with electrolytic lesions of the perirhinal cortex display a deficit in the initial stages of acquisition of the water- 


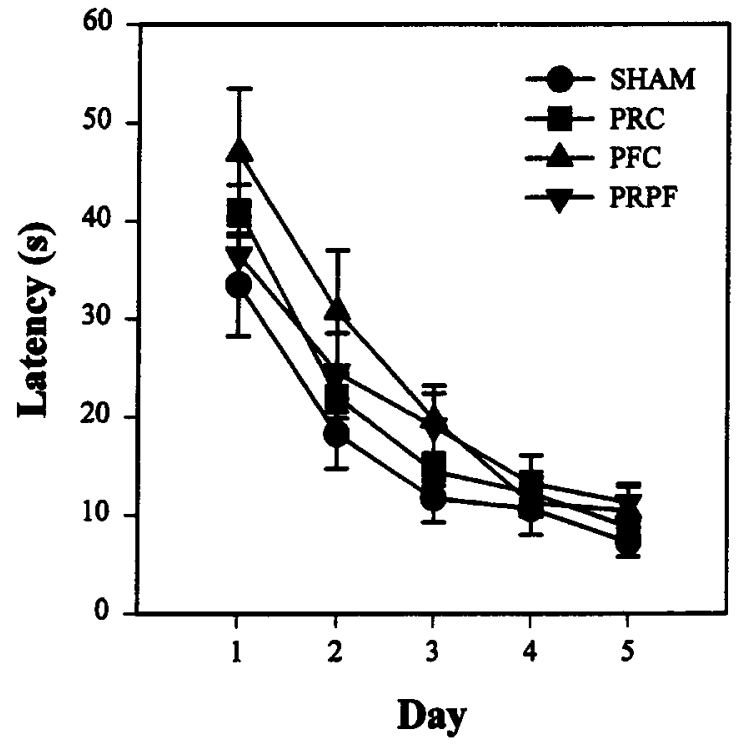

Figure 2. Mean latencies for sham control (SHAM), perirhinal (PRC), prefrontal (PFC), and combined perirhinal-prefrontal (PRPF) cortex-lesioned rats to escape to the platform during place navigation acquisition. PRC- and PFC-lesioned animals displayed a significant deficit in initial acquisition. Each data point represents the mean of eight trials per day (error bars $=$ SEM).

maze task (Liu \& Bilkey, 1998c; Wiig \& Bilkey, 1994a). Furthermore, rats with lesions of the perirhinal cortex displayed a delay-dependent deficit in a probe trial conducted at a short interval after a series of training trials. PRC-lesioned rats were also impaired during the initial stages of a platform reversal task conducted in the water maze, and they displayed a significant deficit in performance in the radial-arm maze. These findings are consistent with previous data from our lab (Liu \& Bilkey, 1998a, 1998b, 1998c; Wiig \& Bilkey, 1994a) and with the proposal that the temporal neocortex regions may be involved in spatial memory processes (Ploner et al., 1999; cf. Bussey, Muir, \& Aggleton, 1999), possibly via interactions with the hippocampus (Gaffan, 1998).

Lesions of the prefrontal cortex produced deficits in water-maze acquisition that were similar to those produced by lesions of the perirhinal cortex. PFC-lesioned rats also displayed a deficit in a reversal procedure conducted in the water maze and in a radial-maze procedure. This finding is consistent with the hypothesis that the prefrontal region is involved in working-memory processes that require the maintenance and manipulation of memory representations in their activated state (e.g., Goldman-Rakic, 1995; Miller et al., 1996; Shimamura, 1995 ) and with the results of several previous studies of the effects of prefrontal lesions on spatial tasks. It has been shown, for example, that such lesions disrupt the processing of egocentric spatial information (Kesner, 1989) and disrupt a spatial delayed nonmatching-to-sample procedure (Kesner, Hunt, Williams, \& Long, 1996). It has also been reported that prefrontal-hippocampal interac- tions are required for delayed performance in the radial maze (Seamans, Floresco, \& Phillips, 1998). In contrast to the current findings, however, de Bruin, SanchezSanted, Heinsbroek, Donker, and Postmes (1994) reported that similarly located dorsomedial prefrontal lesions did not affect acquisition or probe trials in the standard water maze. It is unclear what factors account for this difference; however, it should be noted that there was variation between the two studies in terms of the lesion method, the intertrial interval, and the time that the rats were left on the platform during training.

If a primary function of the prefrontal cortex involves accessing memory representations that may, in part, reside in the PRC, one would expect that the effects of combined lesions of these two structures should be at least equivalent to the maximal effects of lesions to one of the structures alone. This was indeed the case for the platform reversal test (where the combined lesion group performed worse than did either of the single-lesion groups) and in the radial-maze task (where the performance of the combined group was similar to that of the single-lesion groups). Surprisingly, however, the performance of rats with combined PRC and PFC lesions was significantly better during the water-maze probe than the performance of rats with PRC lesions alone. This pattern of findings suggests that although the prefrontal cortex may contribute to working-memory processes under some conditions, the relationship between the prefrontal and temporal regions is not straightforward. Rather, this pattern of findings may reflect the differential participation of the prefrontal cortex in executive processes in some tasks-

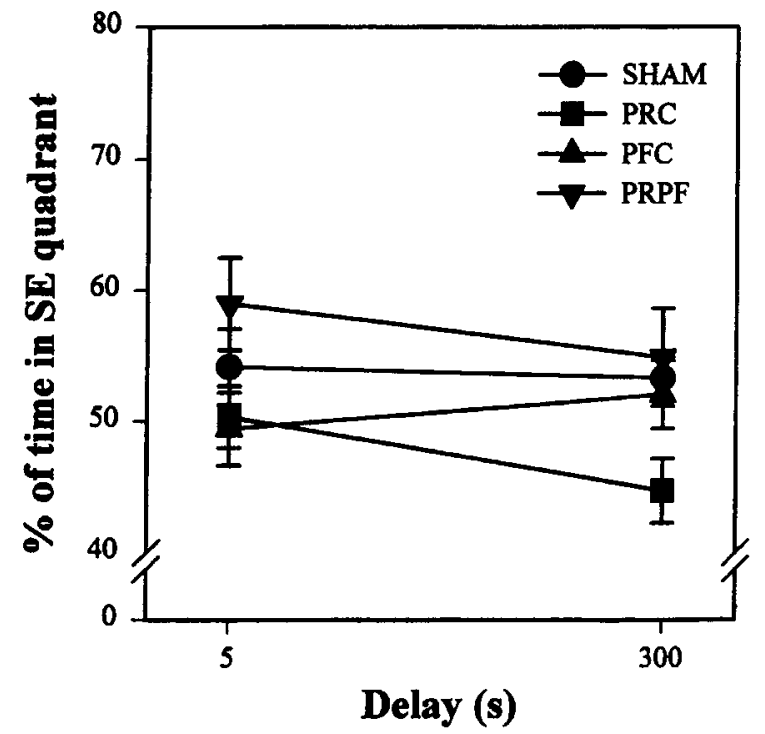

Figure 3. The mean percentage of time spent in the target quadrant (SE) for sham control (SHAM), perirhinal (PRC), prefrontal (PFC), and combined perirhinal-prefrontal (PRPF) cortex-lesioned rats during a probe test that followed four training trials by either 5 or 300 sec. Rats with PRPF lesions performed significantly better at the 300 -sec delay than did rats with PRC lesions alone. 


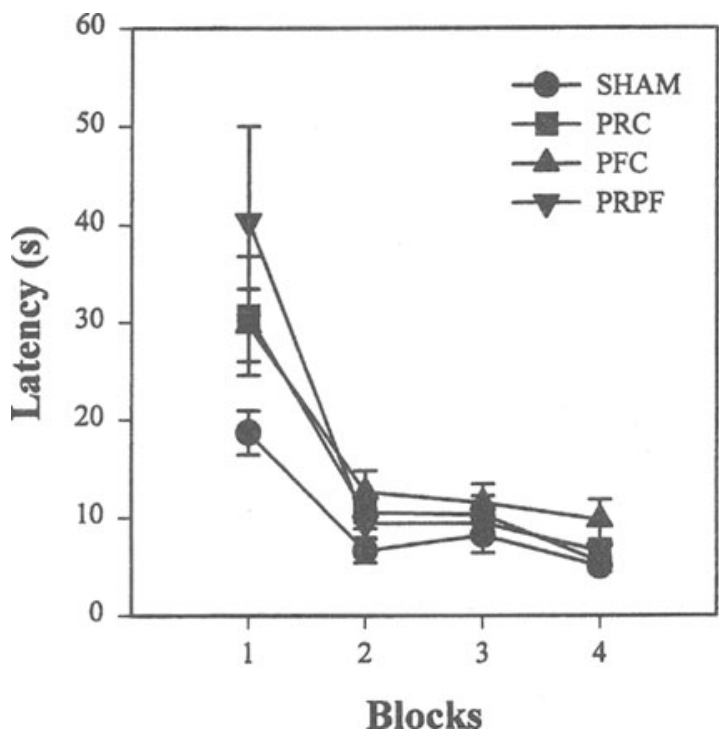

Figure 4. Mean latencies for sham control (SHAM), perirhinal (PRC), prefrontal (PFC), and combined perirhinal-prefrontal (PRPF) cortex lesioned rats to escape to the platform during reversal of the place navigation test. Each data point represents the mean of a block of four trials (there were eight trials per day). All lesioned rats had significantly longer latencies during Block 1.

in, for example, the process by which an appropriate search strategy is selected in the water maze.

Rats can use one or more of several different search strategies in order to navigate to the hidden platform in the water-maze task. These strategies include piloting (Prados \& Trobalon, 1998), path integration (Benhamou, 1997; McNaughton, Chen, \& Markus, 1991; Save, 1997; Whishaw, McKenna, \& Maaswinkel, 1997), and beacon homing (this may have occurred in Eichenbaum, Stewart, \& Morris, 1990). It is also apparent that rats will adopt search strategies that result in an increase in path sinuosity, thereby intensifying the search around the target, once they are near the platform location. This type of strategy appears to be a form of "area-concentrated" search, which has been described previously in ethological studies of foraging and homing behavior (e.g., Benhamou, 1994).

It is likely that the commonly used measures of watermaze performance (latency and time in quadrant-TIQ) may not be equally responsive to changes in these different types of search strategy. For example, it is possible that the TIQ measure, because of its focus on behavior that occurs around the platform location, might be more sensitive to alterations in the area-concentrated search than to changes in other search strategies. In contrast, latency may be a relatively insensitive measure of changes in the area-concentrated strategy. It is, therefore, important to note that although the PRC-lesioned rats did not display a deficit in the latency measure during the final days of training, or an abnormal latency to their first crossing over the previous position of the platform during the probe test, their TIQ measure in the probe trial was significantly reduced at the longer delay interval.

A latency/TIQ dissociation has been described previously in rats with fornix/fimbria (Eichenbaum et al., 1990) and entorhinal/subicular (Schenk \& Morris, 1985) lesions, and it has previously been proposed that this reflects the difference between a task (getting to the platform from the starting position) that can be solved by a relatively inflexible "procedural" (or habit-based) memory system (e.g., using beacon homing), and a task (searching for the platform at the previous location) that requires flexible "declarative" memory (e.g., using path integration or piloting). This proposal is supported by reports of stereotyped behavior in fornix/fimbria- (Eichenbaum et al., 1990) and entorhinal/subicular- (Schenk \& Morris, 1985) lesioned rats. In the present study, however, an analysis of the paths of the PRC-lesioned rats on the final day of training (at which point, latency measures were virtually identical to sham rats) did not support the proposition that they utilized an overly inflexible, stereotyped strategy.

An alternative explanation of the latency/TIQ dissociation can be generated, however, by focusing on the extent to which different search strategies are likely to be differentially dependent on object memory. This explanation proposes that when a rat is well trained in the water-maze task it generates its initial path toward the hidden platform with location as a goal (i.e., a "location-targeted" search; cf. "getting there," Whishaw, Cassel, \& Jarrard, 1995). Since generating a path to a location does not require access to information about the object properties (e.g., shape, texture, color) of the target, this strategy is unlikely to

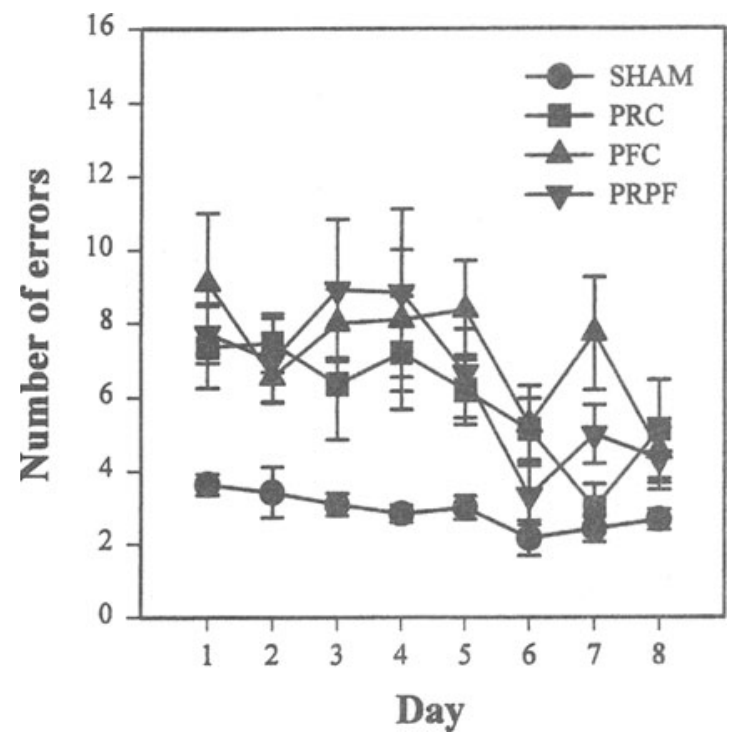

Figure 5. Mean number of errors made in the standard radialarm maze working-memory task for sham control (SHAM), perirhinal (PRC), prefrontal (PFC), and combined perirhinalprefrontal (PRPF) cortex lesioned rats during the training period. All lesioned rats made significantly more errors than did SHAM controls during the initial 5 days of training. 
require access to object recognition memory. As long as this location targeted process is intact, latency measures in the water maze should be normal. Furthermore, it is also possible that an animal that repetitively utilizes this location-targeted strategy in a probe trial could generate normal TIQ scores. Once a normal rat reaches the region near where the platform is (or was) located, however, information about the object properties of the target (e.g., the tactile properties of the platform) would normally become critical for target recognition. This would require that the rat access a prospective memory representation of the target (which could include a reference to location; see, e.g., Kahneman, Treisman, \& Gibbs, 1992; Leslie, Xu, Tremoulet, \& Scholl, 1998) and search for a match. It is possible that the initiation of this "object-targeted" search strategy corresponds to the onset of area-concentrated search behavior. Since PRC lesions have been shown to increase the rate of forgetting of object-related information (Buckley et al., 1997; Buffalo, Reber, \& Squire, 1998; Meunier et al., 1993; Murray, 1996; Wiig \& Bilkey, 1995; Zola-Morgan et al., 1989), such lesions could reduce the duration of the area-concentrated search and so produce the delay-dependent reduction in the TIQ measure that was observed in this and previous studies (Liu \& Bilkey, 1998c).

If it is only the area-concentrated search that is affected by the PRC lesion, an animal that had a damaged PRC, but that was not switched into this object-targeted strategy, might actually benefit in terms of TIQ scores. Therefore, if PFC lesions reduce the probability that a switch will occur between location-targeted and object-targeted searches as a result of an alteration in executive processes, the particular pattern of results that were observed in the present study might emerge. That is, PRC-lesioned rats would display a reduction in TIQ because they were actively switched into a search strategy that was dependent on a dysfunctional object memory. In contrast, PFClesioned and combined-lesion rats could continue to use the location-targeted strategy to repeatedly approach the platform location and generate normal TIQ measures (and hence the apparent amelioration of the PRC-lesion effects in the combined-lesion animals).

The general nature of the explanation described above is consistent with the proposal that the prefrontal cortex is involved in executive processes (Baddeley, 1996; Baddeley \& Della Sala, 1996; Owen, Morris, Sahakian, Polkey, \& Robbins, 1996), such as the switching of retrieval plans (Baddeley, 1996). Furthermore, Kesner (1989) has previously shown that rats with medial prefrontal lesions display difficulty in shifting strategies from a retrospective to a prospective memory code, and a number of human studies indicate that frontal damage produces a deficit in set switching, as evidenced by problems with tests such as the Wisconsin card sorting task (Ridley, 1994). This account is also consistent with the results of a recent imaging study, wherein it was reported that, in normal human subjects, frontal regions are activated when strategy switching is required in a spatial navigation task (Maquire et al., 1998).
Although the proposed model is somewhat speculative, it presents hypotheses regarding swim patterns and search strategies that can be tested in future studies. Further research will, however, be required in order to determine why the lesion-induced amelioration effect was specific to the water-maze probe task. The fact that the effect did not occur in the water-maze reversal, or in the radial-maze procedure, may be the result of the rat's use of different search strategies during the acquisition of a procedure, as opposed to the performance of that procedure once it has become well learned. Furthermore, it is also possible that rats use different strategies in the appetitive radial- or plus-maze task than in the aversive water-maze procedure (Dudchenko, Goodridge, Seiterle, \& Taube, 1997; Martin, Harley, Smith, Hoyles, \& Hynes, 1997).

It should be noted that the overall magnitude of the PRC-lesion effect in the water-maze probe is relatively mild compared with the effects observed in the radial maze and in a delayed nonmatching-to-position procedure conducted in the T maze (Liu \& Bilkey, 1998a). This may reflect a differential involvement of the PRC in the rapid acquisition and storage of trial-dependent information (radial maze and T maze), as opposed to the storage of trial-uniform "reference memory" information (water maze). In this vein, Wise and Murray (1999) have recently proposed that the hippocampus and surrounding cortex are involved in the rapid acquisition of arbitrary visuomotor mappings. This account emphasizes the rapid formation of these mappings and suggests that learning can also occur via other brain systems if repeated training is available. This might explain why the PRC lesion induced a deficit in rats in the present study only during the initial stages of training in the water maze, in the first few trials of the reversal, and in the probe trial when the probe occurred shortly after a "reminder" trial. It may also explain why one recent study determined that PRC lesions did not produce a deficit in a delayed matching-to-place (DMTP) procedure in the water maze (Glenn \& Mumby, 1998). A key factor in the latter study may be that rats received a large number of training trials to each platform position (208 swims distributed across 10 platform positions with the first 144 training swims conducted with delay intervals $30 \mathrm{sec}$ or less that were unlikely to place demands on PRC-dependent memory) before the DMTP data were gathered. This pretraining may have provided an opportunity for the animals to learn to perform the task via non-PRC mechanisms. In contrast, recent data from our laboratory indicate that PRC lesions do produce a delay-dependent deficit in this type of DMTP task when rats are tested with minimal pretraining (Liu \& Bilkey, 1999b).

Bussey et al. (1999) have recently reported that rats with lesions that included the PRC showed no impairments in a radial-maze and standard water-maze task. It is not clear why this group's data differ from our present and previous findings; however, we have previously argued that the radial-maze habituation procedure utilized by this group may have provided some measure of pretraining and, 
hence, allowed for the development of PRC-independent strategies (Liu \& Bilkey, 1999a). It is less clear why these researchers did not find a deficit in the water maze; although, given the findings above, it is possible that differences in the rat strain, the number of trials per session, the time spent on the platform, and/or the intertrial interval may have been critical. Further research will, however, be required in order to ascertain the key factor(s). This aside, however, we believe that our results are consistent with the proposal that the temporal neocortical regions are involved in some form of spatial memory process (Ploner et al., 1999). One possibility may involve the melding of object and place information via interactions with the hippocampus (Gaffan, 1998).

In summary, the present study provides a comparison of the effects of PRC and PFC lesions on the performance of spatial memory tasks. In most cases, the effects of PRC and PFC lesions were similar, in support of a model that proposes that the prefrontal region is involved in workingmemory processes that involve the maintenance and manipulation of memory representations in their activated state (see, e.g., Goldman-Rakic, 1995; Miller et al., 1996; Shimamura, 1995). The effects of combined lesions of these structures were, however, inconsistent with the view that this is the only function that this region serves. Rather, the latter results are better viewed as reflecting a more complex role of the PFC in executive function, whereby the PFC lesion may also alter the strategies that a rat uses to locate the platform in the water-maze probe. A recent study suggests that these working memory and executive processes can be dissociated in humans according to lesion location (Bechara, Damasio, Tranel, \& Anderson, 1998). It remains to be seen whether a similar dissociation can be generated in the rat by restricting lesions to subareas of the PFC.

\section{REFERENCES}

BADDELEY, A. (1986). Working memory. Oxford: Oxford University Press, Clarendon Press.

Baddeley, A. (1996). The fractionation of working memory. Proceedings of the National Academy of Sciences, 93, 13468-13472.

Baddeley, A., \& Della Sala, S. (1996). Working memory and executive control. Philosophical Transactions of the Royal Society of London: Series B, 351, 1397-1403.

Bechara, A., Damasio, H., Tranel, D., \& Anderson, S. W. (1998). Dissociation of working memory from decision making within the human prefrontal cortex. Journal of Neuroscience, 18, 428-437.

Benhamou, S. (1994). Spatial memory and searching efficiency. Animal Behaviour, 47, 1423-1433.

Benhamou, S. (1997). Path integration by swimming rats. Animal Behaviour, 54. 321-327.

Buckley, M. J., GafFan, D., \& Murray, E. A. (1997). Functional double dissociation between two inferior temporal areas: Perirhinal cortex versus middle temporal gyrus. Journal of Neurophysiology, 77, 587-598.

Buffalo, E. A., Reber, P. J., \& SQuire, L. R. (1998). The human perirhinal cortex and recognition memory. Hippocampus, 8, 330-339.

Burwell, R. D., Witter, M. P., \& Amaral, D. G. (1995). Perithinal and postrhinal cortices of the rat: A review of the neuroanatomical literature and comparison with findings from the monkey brain. Hippocampus, 5, 390-408.
Bussey, T. J., Muir, J. L., \& AGgleton, J. P. (1999). Functionally dissociating aspects of event memory: The effects of combined perirhinal and postrhinal cortex lesions on object and place memory in the rat. Journal of Neuroscience, 19, 495-502.

de Bruin, J. P. C., Sanchez-Santed, F., Heinsbroek, R. P. W. Donker, A., \& Postmes, P. (1994). A behavioural analysis of rats with damage to the medial prefrontal cortex using the Morris water maze: Evidence for behavioural flexibility, but not for impaired spatial navigation. Brain Research, 652, 323-333.

Dudchenko, P. A., Goodridge, J. P., Seiterle, D. A., \& Taube, J. S. (1997). Effects of repeated disorientation on the acquisition of spatial tasks in rats: Dissociation between the appetitive radial-arm maze and aversive water maze. Journal of Experimental Psychology: Animal Behavior Processes, 23, 194-210.

Eichenbaum, H., Stewart, C., \& Morris, R. G. M. (1990). Hippocampal representation in spatial learning. Journal of Neuroscience, 10, 331-339.

Ennaceur, A., Neave, N., \& Aggleton, J. P. (1996). Neurotoxic lesions of the perirhinal cortex do not mimic the behavioural effects of fornix transection in the rat. Behavioural Brain Research, 80, 9-25.

Funahashi, S., INOUE, M., \& Kubota, K. (1997). Delay-period activity in the primate prefrontal cortex encoding multiple spatial positions and their order of presentation. Behavioural Brain Research, 84, 203-223.

Fuster, J. M., Bauer, R. H., \& Jervey, J. P. (1985). Functional interactions between inferotemporal and prefrontal cortex in a cognitive task. Brain Research, 330, 299-307.

GaFFAN, D. (1998). Ideothetic input into object-place configuration as the contribution to memory of the monkey and human hippocampus: A review. Experimental Brain Research, 123, 201-209.

GlenN, M. J., \& Mumby, D. G. (1998). Place memory is intact in rats with perirhinal cortex lesions. Behavioral Neuroscience, 112, 13531365.

Goldman, P. S., \& Rosvold, H. E. (1970). Localisation of function within the dorsolateral prefrontal cortex of the rhesus monkey. Experimental Neurology, 27, 291-304.

Goldman-RaKIC, P. S. (1995). Cellular basis of working memory. Neuron, 14, 477-485.

Granon, S., Vidal, C., Thinus-Blanc, C., Changeux, J., \& Poucet, B. (1994). Working memory, response selection, and effortful processing in rats with medial prefrontal lesions. Behavioral Neuroscience, 108, 883-891.

Gutnikov, S. A., MA, Y. Y., \& Gaffan, D. (1997). Temporo-frontal disconnection impairs visual-visual paired association learning but not configural learning in Macaca monkeys. European Journal of Neuroscience, 9, 1524-1529.

Kahneman, D., Treisman, A., \& Gibbs, B. J. (1992). The reviewing of object files: Object-specific integration of information. Cognitive Psychology, 24, 174-219.

KESNER, R. P. (1989). Retrospective and prospective coding of information: Role of the medial prefrontal cortex. Experimental Brain Research, 74, 163-167.

Kesner, R. P., Hunt, M. E., Williams, J. M., \& LonG, J. M. (1996). Prefrontal cortex and working memory for spatial response, spatial location, and visual object information in the rat. Cerebral Cortex, 6 , $311-318$.

KolB, B. (1990). Prefrontal cortex. In B. Kolb \& R. C. Tees (Eds.), The cerebral cortex of the rat (pp. 437-458). London: MIT Press.

Leslie, A. M., Xu, F., Tremoulet, P. D., \& Scholl, B. J. (1998). Indexing and the object concept: Developing "what" and "where" systems. Trends in Cognitive Sciences, 2, 10-18.

LIU, P., \& BILKEY, D. K. (1998a). Excitotoxic lesions centered on perirhinal cortex produce delay-dependent deficits in a test of spatial memory. Behavioral Neuroscience, 112, 512-524.

LIU, P.. \& BiLkEY, D. K. (1998b). Lesions of perirhinal cortex produce spatial memory deficits in the radial maze. Hippocampus, 8, 114-121.

LIU, P., \& BILKEY, D. K. (1998c). Perirhinal cortex contributions to performance in the Morris water maze. Behavioral Neuroscience, 112 , 304-315.

LiU, P., \& BILKEY, D. K. (1999a). The effect of excitotoxic lesions cen- 
tered on perirhinal cortex in two versions of the radial-arm maze. Behavioral Neuroscience, 113, 672-682.

LiU, P., \& BILKEY, D. K. (1999b). Perirhinal cortex lesions produce a delay-dependent deficit in the working memory version of the water maze. Society for Neuroscience Abstracts, 25, 92.

Maguire, E. A., Burgess, N., Donnet, J. G., Franckowiak, R. S. J., FRITH, C. D., \& O'KEEFE, J. (1998). Knowing where and getting there: A human navigation network. Science, 280, 921-924.

Martin, G. M., Harley, C. W., Smith, A. R., Hoyles, E. S., \& Hynes, C. A. (1997). Spatial disorientation blocks reliable goal location on a plus maze but does not prevent goal location in the Morris maze Journal of Experimental Psychology: Animal Behavior Processes, 23, 183-193.

McNaughton, B. L., Chen, L. L., \& Markus, E. J. (1991), "Dead reckoning," landmark learning, and the sense of direction: A neurophysiological and computational hypothesis. Journal of Cognitive Neuroscience, 3, 190-202.

Meunier, M., Bachevalier, J., Mishkin, M., \& Murray, E. A. (1993). Effects on visual recognition of combined and separate ablations of the entorhinal and perirhinal cortex in rhesus monkeys. Journal of Neuroscience, 13, 5418-5432.

Miller, E. K., Erickson, C. A., \& Desimone, R. (1996). Neural mechanisms of visual working memory in prefrontal cortex of the macaque. Journal of Neuroscience, 16, 5154-5167.

Mumby, D. G., \& PiNeL, J. P. J. (1994). Rhinal cortex lesions and object recognition in rats. Behavioral Neuroscience, 108, 11-18.

Murray, E. A. (1996). What have ablation studies told us about the neural substrates of stimulus memory? Seminars in the Neurosciences, 8, 13-22.

Olton, D. S., \& Samuelson, R. J. (1976). Remembrance of places passed: Spatial memory in rats. Journal of Experimental Psychology: Animal Behavior Processes, 2, 97-115.

Olton, D. S., WALKER, J. A., \& GAGE, F. H. (1978). Hippocampal connections and spatial discrimination. Brain Research, 139, 295-308.

OWen, A. M., Morris, R. G., Sahakian, B. J., Polkey, C. E., \& RoBBINS, T. W. (1996). Double dissociations of memory and executive functions in working-memory tasks following frontal lobe excisions, temporal lobe excisions or amygdalo-hippocampectomy in man. Brain, 119, 1597-1615.

PARKer, A., \& GAFFAN, D. (1998). Interaction of frontal and perirhinal cortices in visual object recognition memory in monkeys. European Journal of Neuroscience, 10, 3044-3057.

PAXINOS, G., \& WATSON, C. (1986). The rat brain in stereotaxic coordinates (2nd ed.). San Diego: Academic Press

Ploner, C. J., Gaymard, B. M., Ehrlé, N., Rivaud-Pechoux, S. Baulac, M., Brandt, S. A., Clémenceau, S., Samson, S., \& PierrotDESEILLIGNY, C. (1999). Spatial memory deficits in patients with lesions affecting the medial temporal neocortex. Annals of Neurology, 45, 312-319.

Prados, J., \& Trobalon, J. B. (1998). Locating an invisible goal in a water maze requires at least two landmarks. Psychobiology, 26, 4248 .
RiDLEY, R. M. (1994). The psychology of perseverative and stereotyped behaviour. Progress in Neurobiology, 44, 221-231.

Sanchez-Santed, F., Bruin, J. P. C., Heinsbroek, R. P. W., \& VerWER, R. W. H. (1997). Spatial delayed alternation of rats in a T-maze: Effects of neurotoxic lesions of the medial prefrontal cortex and of T-maze rotations. Behavioural Brain Research, 84, 73-79.

SAVE, E. (1997). The contribution of visual and inertial mechanisms to navigation in total darkness. Animal Learning \& Behavior, 25, 324 334

SCHENK, F., \& Morris, R. G. M. (1985). Dissociation between components of spatial memory after recovery from the effects of retrohippocampal lesions. Experimental Brain Research, 58, 11-18.

Seamans, J. K., Floresco, S. B., \& Phillips, A. G. (1998). D1 receptor modulation of hippocampal-prefrontal cortical circuits integrating spatial memory with executive functions in the rat. Journal of Neuroscience, 18, 1613-1621.

Shaw, C., \& Aggleton, J. P. (1993). The effects of fornix and medial prefrontal lesions on delayed non-matching-to-sample by rats. $B e$ havioural Brain Research, 54, 91-102.

Shimamura, A. P. (1995). Memory and the prefrontal cortex. In J. Grafman, K. J. Holyoak, \& F. Boller (Eds.), Structure and functions of the human prefrontal cortex (Annals of the New York Academy of Sciences, Vol. 769, pp. 151-159). New York: New York Academy of Sciences.

Whishaw, I. Q., CASSEL, J., \& JaRRARD, L. E. (1995). Rats with fimbriafornix lesions display a place response in a swimming pool: A dissociation between getting there and knowing where. Journal of Neuroscience, 15, 5779-5788.

Whishaw, I. Q., McKenna, J. E., \& MaAswinkel, H. (1997). Hippocampal lesions and path integration. Current Opinion in Neurobiology, 7, 228-234.

WiIG, K. A., \& BILKEY, D. K. (1994a). Effects of perirhinal cortex lesions on spatial reference memory in the rat. Behavioural Brain Research, 63, 101-109.

WiIG, K. A., \& BILKeY, D. K. (1994b). Perirhinal cortex lesions in rats disrupt performance in a spatial DNMS task. NeuroReport, 5, 14051408 .

WIIG, K. A., \& BILKEY, D. K. (1995). Lesions of the rat perirhinal cortex exacerbate the memory deficit observed following damage to the fimbria-fornix. Behavioral Neuroscience, 109, 620-630.

Wise, S. P., \& Murray, E. A. (1999). Role of the hippocampal system in conditional motor learning: Mapping antecedents to action. Hippocampus, 9, 101-117.

ZAR, J. H. (1996). Biostatistical analysis (3rd ed.). New Jersey: PrenticeHall.

Zola-Morgan, S., Squire, L. R., Amaral, D. G., \& Suzuki, W. A. (1989). Lesions of perirhinal and parahippocampal cortex that spare the amygdala and hippocampal formation produce severe memory impairment. Journal of Neuroscience, 9, 4355-4370.

(Manuscript received November 24, 1998; revision accepted for publication October 4, 1999.) 